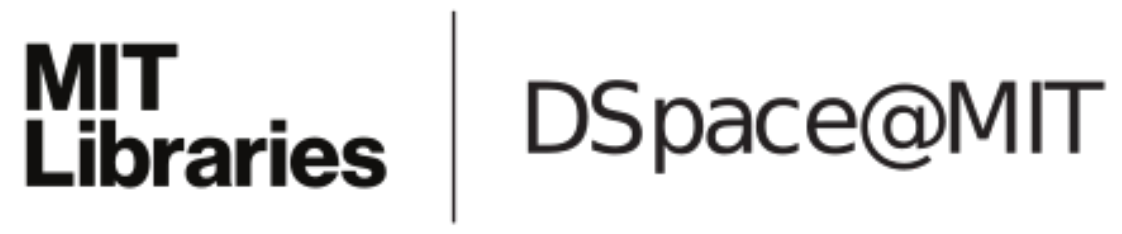

\author{
MIT Open Access Articles
}

The nucleus of Comet 10p/Tempel 2 in 2013 and consequences regarding its rotational state: early science from the Discovery Channel Telescope

The MIT Faculty has made this article openly available. Please share how this access benefits you. Your story matters.

Citation: Schleicher, David G., Matthew M. Knight, and Stephen E. Levine. "The Nucleus of Comet 10p/Tempel 2 In 2013 and Consequences Regarding its Rotational State: Early Science from the Discovery Channel Telescope." The Astronomical Journal 146, no. 5 (October 17, 2013): 137. (C) 2013 The American Astronomical Society

As Published: http://dx.doi.org/10.1088/0004-6256/146/5/137

Publisher: IOP Publishing

Persistent URL: http://hdl.handle.net/1721.1/92738

Version: Final published version: final published article, as it appeared in a journal, conference proceedings, or other formally published context

Terms of Use: Article is made available in accordance with the publisher's policy and may be subject to US copyright law. Please refer to the publisher's site for terms of use. 


\title{
THE NUCLEUS OF COMET 10P/TEMPEL 2 IN 2013 AND CONSEQUENCES REGARDING ITS ROTATIONAL STATE: EARLY SCIENCE FROM THE DISCOVERY CHANNEL TELESCOPE
}

\author{
David G. Schleicher ${ }^{1}$, Matthew M. KNight ${ }^{1,3}$, And STEPhen E. Levine ${ }^{1,2}$ \\ ${ }^{1}$ Lowell Observatory, 1400 W. Mars Hill Road, Flagstaff, AZ 86001, USA; dgs@ lowell.edu \\ ${ }^{2}$ Department of Earth, Atmospheric, and Planetary Sciences, Massachusetts Institute of Technology, Cambridge, MA 02139, USA \\ Received 2013 August 22; accepted 2013 September 10; published 2013 October 17
}

\begin{abstract}
We present new lightcurve measurements of Comet 10P/Tempel 2 carried out with Lowell Observatory's Discovery Channel Telescope in early 2013 when the comet was at aphelion. These data represent some of the first science obtained with this new $4.3 \mathrm{~m}$ facility. With Tempel 2 having been observed to exhibit a small but ongoing spin-down in its rotation period for over two decades, our primary goals at this time were two-fold. First, to determine its current rotation period and compare it to that measured shortly after its most recent perihelion passage in 2010, and second, to disentangle the spin-down from synodic effects due to the solar day and Earth's orbital motion and to determine the sense of rotation, i.e., prograde or retrograde. At our midpoint of 2013 February 24, the observed synodic period is $8.948 \pm 0.001 \mathrm{hr}$, exactly matching the predicted prograde rotation solution based on 2010 results, and yields a sidereal period of the identical value due to the solar and Earth synodic components just canceling out during the interval of the 2013 observations. The retrograde solution is ruled out because the associated sidereal periods in 2010 and 2013 are quite different even though we know that extremely little outgassing, needed to produce torques, occurred in this interval. With a definitive sense of rotation, the specific amounts of spin-down to the sidereal period could be assessed. The nominal values imply that the rate of spin-down has decreased over time, consistent with the secular drop in water production since 1988. Our data also exhibited an unexpectedly small lightcurve amplitude which appears to be associated with viewing from a large, negative sub-Earth latitude, and a lightcurve shape deviating from a simple sinusoid implying a highly irregularly shaped nucleus.
\end{abstract}

Key words: comets: general - comets: individual (10P/Tempel 2) - methods: data analysis methods: observational

Online-only material: color figure

\section{INTRODUCTION}

Comet $10 \mathrm{P} /$ Tempel 2 is the second largest Jupiter-family comet in existence-only $28 \mathrm{P} /$ Neujmin 1 is known to be larger-and is comparable in size to 1P/Halley. Unlike Halley, however, Tempel 2 has a very small fraction of its surface that is still active $(<1 \%)$ and is therefore one of the most highly evolved comets. These two properties have made it possible to study its nucleus at both larger heliocentric distances (Jewitt \& Meech 1988; Mueller \& Ferrin 1996) and at favorable perihelion passages (Jewitt \& Luu 1989; A'Hearn et al. 1989; Wisniewski 1990; Knight et al. 2011, 2012). It also exhibits a strong asymmetry about perihelion in brightness (Sekanina 1979) and production rates due to the seasonal effects associated with the orientation of the rotation axis and a small source region near one pole that dominates the outgassing (Knight et al. 2012). Of particular interest here is that Tempel 2 is one of the first comets to exhibit not just a change in its rotational period (Mueller \& Ferrin 1996) but a progressive spin-down over multiple apparitions. Our lightcurve measurements obtained at the 1999 apparition gave a period of $8.941 \pm 0.002 \mathrm{hr}$, about $32 \mathrm{~s}$ longer than the value of $8.932 \pm 0.001 \mathrm{hr}$ two orbits earlier in 1988 based on a reanalysis of all available data (Knight et al. 2011). We followed this with further measurements in 2010, finding a period of $8.950 \pm 0.002 \mathrm{hr}$, thereby strongly implying the spin-down is caused by progressive torquing associated with the outgassing from the near-polar source region (Knight et al. 2012).

\footnotetext{
3 Visiting scientist at The Johns Hopkins University Applied Physics Laboratory, 11100 Johns Hopkins Road, Laurel, MD 20723, USA.
}

The situation, however, is somewhat more complicated than it first appears. While the measured spin-down was the same between 1988 and 1999, and between 1999 and 2010, the observations in the earlier apparitions were obtained several months prior to perihelion, while the observations in 2010 were obtained between two and six months following perihelion. Thus a somewhat larger change in period was expected in the second pairing if the amount of torque remained the same at every perihelion passage. While our 2010 result might be explained by a secular decrease in outgassing that we observed during the past quarter century, it remained unclear what period Tempel 2 ultimately attained as it retreated from the Sun in 2011 and activity dropped off. Additionally, all of the reported values for its rotation period are synodic rather than sidereal. While some evidence has suggested that Tempel 2 is in prograde rotation (Sekanina 1991), a reanalysis of the same data gave conflicting results (Knight et al. 2011). Since the spin-down of the nucleus adds a further complication to extracting the sense of rotation from varying synodic effects, we considered the direction of rotation as being unresolved, resulting in a pair of possible sidereal periods for each synodic result. For example, the 2010 synodic value of $8.950 \pm 0.002 \mathrm{hr}$ corresponds to a prograde sidereal solution of $8.948 \pm 0.002 \mathrm{hr}$ or a retrograde solution of $8.955 \pm 0.002 \mathrm{hr}$, where these offsets are asymmetric due to Earth's orbital motion. Since the difference between synodic and sidereal varies with orbital position and observer geometries, this results in an added ambiguity to the specific amount of torque delivered each apparition. Fortunately, the sense of rotation can be resolved with additional measurements of Tempel 2 at a more distant location in its orbit when the synodic/ sidereal difference is much smaller than near perihelion. 
Table 1

Summary of Tempel 2 Observational Circumstances in $2013^{\mathrm{a}}$

\begin{tabular}{|c|c|c|c|c|c|c|c|c|c|}
\hline UT Date & UT & $\begin{array}{l}\Delta T^{\mathrm{b}} \\
\text { (day) }\end{array}$ & $\begin{array}{c}r_{\mathrm{H}} \\
(\mathrm{AU})\end{array}$ & $\begin{array}{c}\Delta \\
(\mathrm{AU})\end{array}$ & $\begin{array}{c}\theta \\
\left({ }^{\circ}\right)\end{array}$ & $\begin{array}{l}\Delta m_{\theta}{ }^{\mathrm{c}} \\
(\mathrm{mag})\end{array}$ & $\begin{array}{l}\text { Ecl. Long. } \\
\text { Earth }\left(^{\circ}\right)^{\mathrm{d}}\end{array}$ & $\begin{array}{l}\text { Ecl. Long. } \\
\text { Sun }\left(^{\circ}\right)^{\mathrm{e}}\end{array}$ & Conditions \\
\hline Jan 12 & $3: 34-13: 29$ & +12.4 & 4.697 & 3.770 & 4.5 & 0.42 & 203.8 & 309.3 & Clouds \\
\hline Jan 13 & $3: 35-13: 27$ & +13.4 & 4.698 & 3.765 & 4.3 & 0.41 & 204.1 & 309.4 & Intermittent clouds \\
\hline Mar 11 & $2: 51-10: 38$ & +70.3 & 4.709 & 3.970 & 8.8 & 0.64 & 225.4 & 313.1 & Cirrus \\
\hline Apr 4 & $3: 01-8: 52$ & +94.2 & 4.707 & 4.293 & 11.6 & 0.76 & 234.6 & 314.6 & Cirrus \\
\hline Apr 6 & $2: 56-8: 45$ & +96.2 & 4.706 & 4.323 & 11.8 & 0.76 & 235.5 & 314.8 & Clouds \\
\hline
\end{tabular}

Notes.

a All parameters were taken at the midpoint of each night's observations.

b Time since 2013 January 0.0 .

${ }^{\mathrm{c}}$ Magnitude correction to normalize to $\theta=0^{\circ}$.

${ }^{\mathrm{d}}$ Ecliptic longitude of the Earth as seen from the comet.

e Ecliptic longitude of the Sun as seen from the comet.

With these motivations, and with access to our new $4 \mathrm{~m}$ class Discovery Channel Telescope (DCT), we decided to again measure Tempel 2's rotation period but this time near aphelion. The specific timing of the observations was based not only on the observing geometries but also required waiting for the comet to move sufficiently far away from the galactic plane. The initial night of planned observation also proved to be the first successful night of science operations for DCT, still early in its commissioning phase. We present here lightcurve data obtained over a three month interval, and the resulting derived period along with the implications of this result.

\section{OBSERVATIONS AND REDUCTIONS}

\subsection{Instrumentation}

Lowell Observatory's Discovery Channel Telescope saw first light in 2012 April, less than 7 yr after ground-breaking. The telescope, with a $4.3 \mathrm{~m}$ primary, is located near Happy Jack, AZ, at an elevation of $2337 \mathrm{~m}$ (cf. Levine et al. 2012; Bida et al. 2012). Site testing yielded a median seeing of 0.'84 (Bida et al. 2004) and delivered images already routinely exhibit FWHM of $\sim 0.8$ when atmospheric conditions are good. The first instrument designed for DCT is the Large Monolithic Imager (LMI), which began service in 2012 September. Containing a newly developed $6.1 \mathrm{~K} \times 6.1 \mathrm{~K} \mathrm{CCD}$ by e2v, this camera yields a 12 . 3 on a side field of view at the $f / 6.1$ Ritchey-Chretien focus (Massey et al. 2013). Our observations were obtained using $2 \times 2$ on-chip binning, resulting in a pixel scale of 0.240 , and we used a Kron-Cousins $R$-band filter throughout.

\subsection{Observations and Reductions}

Successful and useful observations were acquired on two nights in 2013 January, one in March, and two in April; planned observations in February were snowed out while limited observations in May were interrupted by technical problems (including a regional power failure) making the remaining data useless for this project. Observational circumstances for each of the five useful nights are given in Table 1, including heliocentric distance $\left(r_{\mathrm{H}}\right)$, geocentric distance $(\Delta)$, and phase angle $(\theta)$. The January and March runs each immediately followed large storms, resulting in quite poor and variable seeing $\left(\sim 1^{\prime \prime} 0-2\right.$ ". 4$)$, while conditions were better in April ( $\left.0^{\prime} .9-11^{\prime \prime} 3\right)$; image quality additionally degraded at high airmasses as we attempted to maximize the temporal coverage each night. As expected during the very early science phase of a new telescope undergoing commissioning, a variety of small issues emerged and some affected the observations. In particular, pointing map problems in January required us to use the sidereal rate and manually guide on stars rather than track at Tempel 2's rate of motion. Fourminute integrations were used, resulting in $1^{\prime \prime} .6$ of trailing of the comet. We were able to use our preferred mode of tracking at the comet's rate of motion on the other runs, resulting in trailed comparison stars, though no tracking was actually required nor performed in April because the comet was within days of its stationary point. With a shorter exposure of $150 \mathrm{~s}$ and the slower rate of motion of the comet as it approached its stationary point, stellar trailing was quite small, 0.'6, in March while the comet trailed by $<0^{\prime \prime} .1$ in April.

The choice of the aperture size for photometric extractions was, as usual, a compromise between a variety of factors, including trailing and seeing as just described, along with the contrast of the object to the sky, and how crowded the field was. The faintness of the comet and the occasional close passage of field stars argued for a small aperture while the other factors pushed for larger sizes, especially in January. Fortunately, as discussed below, no coma was detected and so no removal was necessary. We ultimately chose to minimize the resulting scatter in Tempel 2's lightcurve each night, which resulted in aperture radii of 11 pixels (2".6) in January, 8 pixels (1".9) in March, and 6 pixels (1".4) in April; sky measurements were obtained from the median value in an annulus between 30 and 60 pixels (7".2-14".4). The effects of changing seeing were directly compensated for by using identical apertures for onframe comparison stars (see below), while the differential effects associated with trailing were minimized with these relatively large extraction apertures.

Calibration was performed using standard techniques with the Interactive Data Language software package for removing bias and applying median twilight flat fields. An extinction coefficient of $0.098 \mathrm{mag}$ per unit airmass was determined on January 13, and was applied to all of each nights' data as a first-order correction for airmass. A minimum of seven comparison stars were used each night; the relatively slow motion of the comet at aphelion coupled with the large field of view of LMI meant these stars remained in the field throughout the night. Remaining variations from frame to frame due to cirrus, changing seeing, and second-order airmass effects were compensated for by determining and applying the median value of the deviations of all the comparison stars. In practice, though the resulting standard deviations for the comparison stars were quite small, the scatter for the much fainter comet became unacceptable when this correction was larger than $0.5 \mathrm{mag}$, and we therefore excluded data above this threshold. 


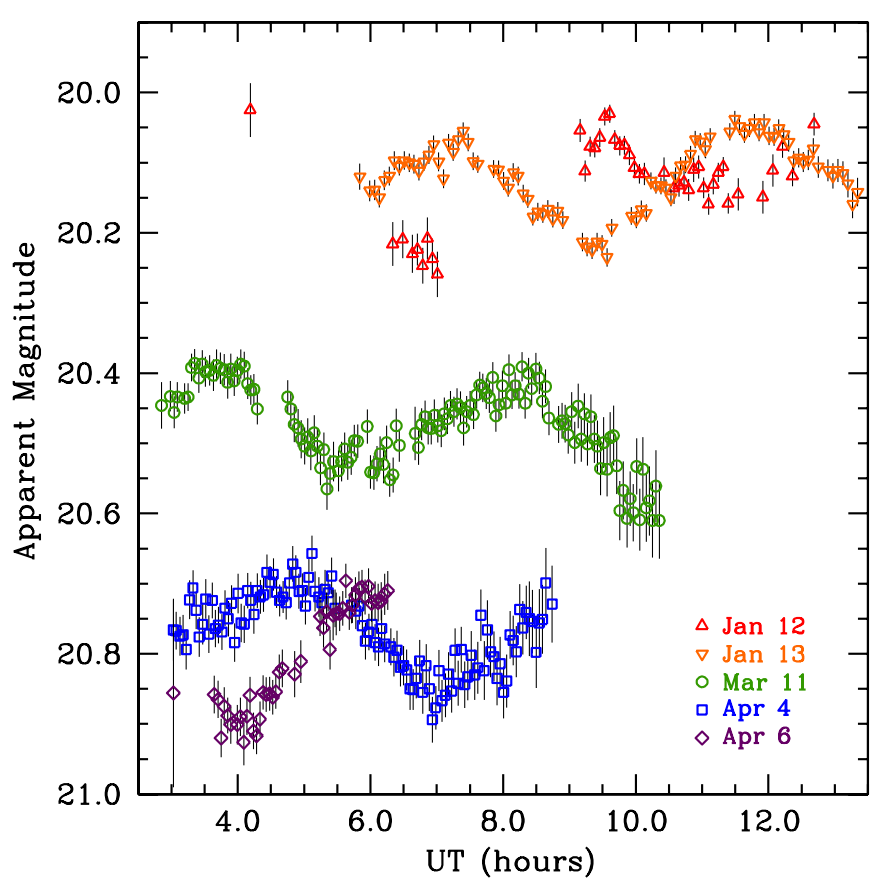

Figure 1. Reduced $R$-band magnitudes $\left(m_{R}\right)$ for our data plotted as a function of UT on each night. The magnitudes have had absolute calibrations, extinction corrections, and comparison star corrections applied and are given in Table 2. The symbols are defined in the legend. Note that while $r_{\mathrm{H}}$ remained approximately constant during the observations, $\Delta$ and $\theta$ increased, causing the brightness to steadily decrease from month to month.

(A color version of this figure is available in the online journal.)

We additionally excluded frames for which the comet was adversely affected by nearby stars, cosmic rays, etc. The uncertainties on each resulting measurement of Tempel 2 were based on the photometric uncertainties combined with the standard deviations of the comparison star corrections.

Absolute flux calibrations were applied by using Sloan Digital Sky Survey (SDSS) $r$ filter catalog values of each of our comparison stars. The average nightly uncertainty in the absolute flux calibrations was $0.06 \mathrm{mag}$, which encompasses both the uncertainty in the catalog magnitudes themselves and color effects in the extinction correction. The resulting nightly instrumental correction varied by as much as 0.3 mag because several nights had no interval when the sky was photometric. While Landolt fields were observed on the few photometric occasions, these measurements were too sparse to significantly improve the absolute calibration but did confirm that our calibration was good to better than $0.1 \mathrm{mag}$. Assuming a solar color, we applied an average offset of $0.22 \mathrm{mag}$ to convert from SDSS $r$ to Cousins $R$. $^{4}$ Final lightcurve measurements and uncertainties of all 422 useful data points (as defined above) are listed in Table 2, as well as the observed mid-times for each image.

\section{LIGHTCURVE RESULTS}

\subsection{Lightcurves}

The reduced lightcurves are displayed in Figure 1 as a function of time for each of the five nights. Immediately evident is the large decrease in brightness during the apparition that will be shown later to be simply due to the combination of increasing distance and increasing phase angle. Gaps in the data on

\footnotetext{
4 http://www.sdss.org/dr5/algorithms/sdssUBVRITransform.html
}

January 12 and the apparent late start on January 13 are due to clouds and/or the comet passing too close to a star. Series of images having the best effective seeing from January 13 and again from April 6 were stacked to search for evidence of a coma; none was detectable on either night. Comparison of the wings of the comet's radial profile to stellar radial profiles on April 4 and April 6 (when the non-sidereal motion was negligible) suggests than any non-stellar component had an upper limit of 5\% of the total cometary flux. Therefore no coma removal was required (or possible) from these observations, unlike observations near perihelion.

To remove the observed secular trend, we next performed a standard asteroidal normalization for these nuclear magnitudes to absolute values by correcting for heliocentric and geocentric distances and to zero phase angle, i.e., from the reduced magnitude, $m_{\mathrm{R}}$, to $m_{\mathrm{R}}(1,1,0)$. Our first attempts at normalization used the same linear phase function that we applied in our previous papers (Knight et al. 2011, 2012). This failed, however, to yield consistent brightnesses among the three observing runs no matter what phase coefficient was used. In retrospect, this was not surprising due to the much smaller phase angles involved in 2013 and the typical asteroidal non-linear opposition effect. Rather than empirically determining the needed curvature, we decided to use a model phase curve that best matched C-type asteroids, those with physical characteristics such as albedo most similar to comet nuclei. Fitting and interpolating the values from Table IV of Bowell \& Lumme (1979), we computed phase adjustments $\left(\Delta m_{\theta}\right)$ for each night as given in Table 1. The resulting $m_{\mathrm{R}}(1,1,0)$ values at lightcurve maxima were in remarkable agreement, indicating that the phase function for Tempel 2's nucleus is indeed well-represented by C-type asteroids, and no further adjustments were applied.

\subsection{Rotation Period}

We continue to use our interactive period search routine for period determinations. As described in more detail in Knight et al. (2011), phase plots are instantaneously updated as we systematically step through possible periods and examine by eye the tightness of resulting lightcurves. Data are color-coded by date, making it easy to see small offsets in either brightness or phase throughout the phased curve. This is particularly useful when the intrinsic scatter due to photometric uncertainties is larger than usual, as we have here; a slightly incorrect period will only have a very slight increase in scatter and most period search algorithms will not "see" that one day is offset from another. In fact, we also used the phase dispersion minimization method (Stellingwerf 1978) and, while this gave the same result, its uncertainty is much greater than what can be easily estimated from the color-coded phase plots, i.e., when clear shifts between observing runs is evident. Note that standard routines can also misinterpret changes in the shape of the lightcurve, another factor here.

Our overall best observed period solution was $8.948 \pm$ $0.001 \mathrm{hr}$, and the corresponding phase plot is shown in the top panel of Figure 2. Since there is no obvious preferred zero point for phasing, we choose 2013 January 0.0 as this placed zero phase away from interesting features and the $\Delta T$ values correspond to the day of the year; prior to phasing, the $\Delta T$ values were first corrected for light travel time from the observed mid-times in Table 1. As with previous apparitions, we have a double-peaked light curve where the two maxima are nearly identical in brightness but the minima are quite different, with the deeper minimum nearly "V"-shaped while the smaller 
Table 2

Photometry of Comet Tempel 2 in 2013

\begin{tabular}{|c|c|c|c|c|c|c|c|c|c|c|c|c|c|c|c|}
\hline Date $^{\mathrm{a}}$ & $\mathrm{UT}^{\mathrm{b}}$ & $m_{R}^{\mathrm{c}}$ & $\sigma_{m_{R}}{ }^{\mathrm{d}}$ & Date $^{a}$ & $\mathrm{UT}^{\mathrm{b}}$ & $m_{R}{ }^{\mathrm{c}}$ & $\sigma_{m_{R}}{ }^{\mathrm{d}}$ & Date $^{a}$ & $\mathrm{UT}^{\mathrm{b}}$ & $m_{R}{ }^{\mathrm{c}}$ & $\sigma_{m_{R}}^{\mathrm{d}}$ & Date $^{a}$ & $\mathrm{UT}^{\mathrm{b}}$ & $m_{R}{ }^{\mathrm{c}}$ & $\sigma_{m_{R}}{ }^{\mathrm{d}}$ \\
\hline Jan 12 & 4.188 & 20.025 & 0.039 & Jan 13 & 7.857 & 20.109 & 0.013 & Mar 11 & 2.983 & 20.433 & 0.022 & Mar 11 & 7.064 & 20.482 & 0.023 \\
\hline Jan 12 & 6.337 & 20.216 & 0.031 & Jan 13 & 7.931 & 20.110 & 0.013 & Mar 11 & 3.040 & 20.456 & 0.023 & Mar 11 & 7.112 & 20.458 & 0.023 \\
\hline Jan 12 & 6.486 & 20.209 & 0.027 & Jan 13 & 8.005 & 20.126 & 0.012 & Mar 11 & 3.090 & 20.434 & 0.021 & Mar 11 & 7.161 & 20.465 & 0.022 \\
\hline Jan 12 & 6.635 & 20.230 & 0.027 & Jan 13 & 8.079 & 20.136 & 0.013 & Mar 11 & 3.199 & 20.436 & 0.022 & Mar 11 & 7.209 & 20.446 & 0.021 \\
\hline Jan 12 & 6.710 & 20.224 & 0.023 & Jan 13 & 8.153 & 20.114 & 0.013 & Mar 11 & 3.250 & 20.434 & 0.020 & Mar 11 & 7.258 & 20.455 & 0.021 \\
\hline $\operatorname{Jan} 12$ & 6.786 & 20.247 & 0.026 & Jan 13 & 8.226 & 20.119 & 0.013 & Mar 11 & 3.300 & 20.392 & 0.020 & Mar 11 & 7.307 & 20.444 & 0.022 \\
\hline Jan 12 & 6.861 & 20.208 & 0.030 & Jan 13 & 8.301 & 20.144 & 0.013 & Mar 11 & 3.350 & 20.386 & 0.020 & Mar 11 & 7.355 & 20.449 & 0.023 \\
\hline Jan 12 & 6.936 & 20.237 & 0.029 & Jan 13 & 8.374 & 20.152 & 0.012 & Mar 11 & 3.413 & 20.407 & 0.021 & Mar 11 & 7.403 & 20.478 & 0.025 \\
\hline Jan 12 & 7.011 & 20.259 & 0.032 & Jan 13 & 8.448 & 20.177 & 0.013 & Mar 11 & 3.464 & 20.387 & 0.020 & Mar 11 & 7.451 & 20.455 & 0.023 \\
\hline Jan 12 & 9.159 & 20.054 & 0.016 & Jan 13 & 8.522 & 20.170 & 0.013 & Mar 11 & 3.514 & 20.398 & 0.021 & Mar 11 & 7.503 & 20.446 & 0.021 \\
\hline Jan 12 & 9.235 & 20.112 & 0.015 & Jan 13 & 8.597 & 20.173 & 0.013 & Mar 11 & 3.576 & 20.396 & 0.022 & Mar 11 & 7.552 & 20.459 & 0.021 \\
\hline Jan 12 & 9.309 & 20.077 & 0.014 & Jan 13 & 8.676 & 20.166 & 0.012 & Mar 11 & 3.630 & 20.403 & 0.023 & Mar 11 & 7.600 & 20.431 & 0.020 \\
\hline Jan 12 & 9.384 & 20.079 & 0.014 & Jan 13 & 8.750 & 20.180 & 0.012 & Mar 11 & 3.681 & 20.389 & 0.023 & Mar 11 & 7.648 & 20.417 & 0.019 \\
\hline Jan 12 & 9.458 & 20.064 & 0.013 & Jan 13 & 8.824 & 20.169 & 0.013 & Mar 11 & 3.736 & 20.393 & 0.022 & Mar 11 & 7.696 & 20.421 & 0.020 \\
\hline Jan 12 & 9.533 & 20.034 & 0.013 & Jan 13 & 8.898 & 20.182 & 0.013 & Mar 11 & 3.793 & 20.395 & 0.022 & Mar 11 & 7.746 & 20.429 & 0.022 \\
\hline Jan 12 & 9.607 & 20.030 & 0.013 & Jan 13 & 9.195 & 20.213 & 0.013 & Mar 11 & 3.846 & 20.413 & 0.023 & Mar 11 & 7.795 & 20.435 & 0.022 \\
\hline $\operatorname{Jan} 12$ & 9.681 & 20.067 & 0.013 & Jan 13 & 9.268 & 20.220 & 0.013 & Mar 11 & 3.895 & 20.394 & 0.022 & Mar 11 & 7.843 & 20.406 & 0.022 \\
\hline $\operatorname{Jan} 12$ & 9.756 & 20.076 & 0.014 & Jan 13 & 9.342 & 20.224 & 0.014 & Mar 11 & 3.945 & 20.411 & 0.022 & Mar 11 & 7.891 & 20.461 & 0.023 \\
\hline Jan 12 & 9.830 & 20.075 & 0.013 & Jan 13 & 9.416 & 20.213 & 0.013 & Mar 11 & 3.994 & 20.396 & 0.020 & Mar 11 & 7.939 & 20.445 & 0.023 \\
\hline Jan 12 & 9.905 & 20.089 & 0.015 & Jan 13 & 9.490 & 20.216 & 0.013 & Mar 11 & 4.045 & 20.387 & 0.021 & Mar 11 & 7.990 & 20.418 & 0.022 \\
\hline Jan 12 & 9.979 & 20.107 & 0.015 & Jan 13 & 9.564 & 20.235 & 0.013 & Mar 11 & 4.098 & 20.390 & 0.021 & Mar 11 & 8.039 & 20.443 & 0.021 \\
\hline Jan 12 & 10.055 & 20.116 & 0.014 & Jan 13 & 9.638 & 20.193 & 0.013 & Mar 11 & 4.147 & 20.415 & 0.022 & $\mathrm{Ma}$ & 8.087 & 20.3 & 0.022 \\
\hline Jan 12 & 10.130 & 20.116 & 0.016 & Jan 13 & 9.934 & 20.176 & 0.013 & Mar 11 & 4.196 & 20.424 & 0.021 & Mar 11 & 8.135 & 20.431 & 0.020 \\
\hline Jan 12 & 10.428 & 20.114 & 0.022 & Jan 13 & 10.008 & 20.181 & 0.012 & Mar 11 & 4.244 & 20.423 & 0.022 & Mar 11 & 8.184 & 20.418 & 0.020 \\
\hline Jan 12 & 10.576 & 20.136 & 0.023 & Jan 13 & 10.082 & 20.167 & 0.013 & Mar 11 & 4.294 & 20.451 & 0.022 & Mar 11 & 8.237 & 20.430 & 0.021 \\
\hline Jan 12 & 10.651 & 20.132 & 0.016 & Jan 13 & 10.156 & 20.172 & 0.012 & Mar 11 & 4.751 & 20.434 & 0.024 & Mar 11 & 8.285 & 20.391 & 0.021 \\
\hline Jan 12 & 10.725 & 20.128 & 0.017 & Jan 13 & 10.230 & 20.126 & 0.012 & Mar 11 & 4.801 & 20.451 & 0.025 & $\mathrm{Ma}$ & 8.334 & 20.4 & 0.022 \\
\hline Jan 12 & 10.800 & 20.139 & 0.016 & Jan 13 & 10.304 & 20.132 & 0.012 & Mar 11 & 4.852 & 20.473 & 0.025 & Mar 11 & 8.382 & 20.4 & 0.022 \\
\hline Jan 12 & 10.873 & 20.110 & 0.017 & Jan 13 & 10.380 & 20.133 & 0.012 & Mar 11 & 4.907 & $20.4^{7}$ & 0.027 & & 8.430 & & 0.024 \\
\hline $\operatorname{Jan} 12$ & 10.948 & 20.106 & 0.016 & Jan 13 & 10.454 & 20.136 & 0.012 & Mar 11 & 4.956 & 20.494 & 0.027 & Mar 11 & 8.496 & 20.394 & 0.023 \\
\hline Jan 12 & 11.022 & 20.136 & 0.015 & Jan 13 & 10.528 & 20.150 & 0.012 & Mar 11 & 5.005 & 20.504 & 0.026 & Mar 11 & 8.545 & 20.407 & 0.023 \\
\hline Jan 12 & 11.096 & 20.159 & 0.015 & Jan 13 & 10.601 & 20.119 & 0.012 & Mar 11 & 5.054 & 20.491 & 0.027 & Mar 11 & 8.593 & 20.440 & 0.024 \\
\hline Jan 12 & 11.170 & 20.131 & 0.014 & Jan 13 & 10.675 & 20.104 & 0.012 & Mar 11 & 5.102 & 20.511 & 0.027 & Mar 11 & 8.641 & 20.419 & 0.024 \\
\hline Jan 12 & 11.245 & 20.114 & 0.014 & Jan 13 & 10.750 & 20.103 & 0.012 & Mar 11 & 5.151 & 20.485 & 0.025 & Mar 11 & 8.689 & 20.464 & 0.025 \\
\hline Jan 12 & 11.319 & 20.106 & 0.014 & Jan 13 & 10.824 & 20.088 & 0.012 & Mar 11 & 5.199 & 20.504 & 0.025 & Mar 11 & 8.837 & 20.473 & 0.026 \\
\hline Jan 12 & 11.394 & 20.158 & 0.015 & Jan 13 & 10.898 & 20.067 & 0.012 & Mar 11 & 5.248 & 20.535 & 0.024 & Mar 11 & 8.886 & 20.468 & 0.026 \\
\hline Jan 12 & 11.544 & 20.145 & 0.023 & Jan 13 & 10.972 & 20.070 & 0.012 & Mar 11 & 5.296 & 20.509 & 0.025 & Mar 11 & 8.935 & 20.472 & 0.027 \\
\hline Jan 12 & 11.916 & 20.149 & 0.023 & Jan 13 & 11.046 & 20.081 & 0.012 & Mar 11 & 5.345 & 20.565 & 0.030 & Mar 11 & 8.983 & 20.488 & 0.027 \\
\hline Jan 12 & 12.065 & 20.111 & 0.023 & Jan 13 & 11.121 & 20.063 & 0.012 & Mar 11 & 5.393 & 20.543 & 0.027 & Mar 11 & 9.031 & 20.455 & 0.028 \\
\hline Jan 12 & 12.215 & 20.077 & 0.016 & Jan 13 & 11.417 & 20.056 & 0.012 & Mar 11 & 5.441 & 20.526 & 0.028 & Mar 11 & 9.079 & 20.499 & 0.030 \\
\hline Jan 12 & 12.363 & 20.119 & 0.014 & Jan 13 & 11.490 & 20.038 & 0.012 & Mar 11 & 5.515 & & 0.029 & & 29 & 20.447 & 0.032 \\
\hline $\operatorname{Jan} 12$ & 12.688 & 20.045 & 0.016 & Jan 13 & 11.565 & 20.048 & 0.012 & Mar 11 & 5.564 & 20.526 & 0.028 & Mar 11 & 9.178 & 20.494 & 0.032 \\
\hline Jan 13 & 5.839 & 20.120 & 0.019 & Jan 13 & 11.640 & 20.059 & 0.012 & Mar 11 & 5.612 & 20.508 & 0.023 & Mar 11 & 9.226 & 20.458 & 0.029 \\
\hline Jan 13 & 5.987 & 20.140 & 0.015 & Jan 13 & 11.713 & 20.051 & 0.012 & Mar 11 & 5.660 & 20.527 & 0.025 & Mar 11 & 9.274 & 20.501 & 0.031 \\
\hline Jan 13 & 6.061 & 20.139 & 0.014 & Jan 13 & 11.787 & 20.043 & 0.012 & Mar 11 & 5.708 & 20.520 & 0.025 & Mar 11 & 9.323 & 20.462 & 0.032 \\
\hline Jan 13 & 6.136 & 20.150 & 0.014 & Jan 13 & 11.861 & 20.057 & 0.012 & Mar 11 & 5.758 & 20.496 & 0.022 & Mar 11 & 9.373 & 20.494 & 0.034 \\
\hline Jan 13 & 6.211 & 20.125 & 0.014 & Jan 13 & 11.935 & 20.043 & 0.012 & Mar 11 & 5.806 & 20.497 & 0.024 & Mar 11 & 9.421 & 20.504 & 0.037 \\
\hline Jan 13 & 6.285 & 20.119 & 0.014 & Jan 13 & 12.009 & 20.062 & 0.013 & Mar 11 & 5.951 & 20.476 & 0.024 & Mar 11 & 9.469 & 20.536 & 0.038 \\
\hline Jan 13 & 6.359 & 20.097 & 0.013 & Jan 13 & 12.083 & 20.063 & 0.013 & Mar 11 & 6.002 & 20.541 & 0.024 & Mar 11 & 9.518 & 20.499 & 0.036 \\
\hline Jan 13 & 6.433 & 20.105 & 0.013 & Jan 13 & 12.157 & 20.051 & 0.013 & Mar 11 & 6.050 & 20.542 & 0.024 & Mar 11 & 9.566 & 20.537 & 0.038 \\
\hline Jan 13 & 6.509 & 20.097 & 0.013 & Jan 13 & 12.231 & 20.060 & 0.013 & Mar 11 & 6.099 & 20.529 & 0.025 & Mar 11 & 9.616 & 20.492 & 0.037 \\
\hline Jan 13 & 6.583 & 20.100 & 0.013 & Jan 13 & 12.305 & 20.071 & 0.013 & Mar 11 & 6.147 & 20.515 & 0.025 & Mar 11 & 9.664 & 20.489 & 0.043 \\
\hline Jan 13 & 6.658 & 20.102 & 0.013 & Jan 13 & 12.379 & 20.097 & 0.014 & Mar 11 & 6.195 & 20.530 & 0.027 & Mar 11 & 9.712 & 20.532 & 0.040 \\
\hline Jan 13 & 6.731 & 20.112 & 0.013 & Jan 13 & 12.454 & 20.093 & 0.014 & Mar 11 & 6.243 & 20.499 & 0.024 & Mar 11 & 9.760 & 20.596 & 0.042 \\
\hline Jan 13 & 6.805 & 20.099 & 0.013 & Jan 13 & 12.528 & 20.099 & 0.014 & Mar 11 & 6.292 & 20.552 & 0.024 & Mar 11 & 9.809 & 20.567 & 0.041 \\
\hline Jan 13 & 6.879 & 20.089 & 0.013 & Jan 13 & 12.602 & 20.096 & 0.015 & Mar 11 & 6.340 & 20.545 & 0.025 & Mar 11 & 9.866 & 20.607 & 0.042 \\
\hline Jan 13 & 6.953 & 20.074 & 0.013 & Jan 13 & 12.676 & 20.080 & 0.015 & Mar 11 & 6.388 & 20.475 & 0.024 & Mar 11 & 9.915 & 20.579 & 0.039 \\
\hline Jan 13 & 7.027 & 20.099 & 0.013 & Jan 13 & 12.750 & 20.106 & 0.015 & Mar 11 & 6.437 & 20.503 & 0.023 & Mar 11 & 9.963 & 20.599 & 0.041 \\
\hline Jan 13 & 7.101 & 20.123 & 0.013 & Jan 13 & 12.899 & 20.111 & 0.016 & Mar 11 & 6.675 & 20.486 & 0.038 & & & 20.533 & 0.040 \\
\hline Jan 13 & 7.176 & 20.072 & 0.013 & Jan 13 & 12.973 & 20.119 & 0.018 & Mar 11 & 6.722 & 20.506 & 0.025 & Mar 11 & & 20.609 & 0.044 \\
\hline Jan 13 & 7.250 & 20.085 & 0.013 & Jan 13 & 13.047 & 20.111 & 0.016 & Mar 11 & 6.773 & 20.473 & 0.023 & Mar 11 & 10.109 & 20.537 & 0.046 \\
\hline Jan 13 & 7.323 & 20.067 & 0.012 & Jan 13 & 13.121 & 20.115 & 0.017 & Mar 11 & 6.821 & 20.462 & 0.023 & Mar 11 & 10.157 & 20.592 & 0.048 \\
\hline Jan 13 & 7.398 & 20.055 & 0.012 & Jan 13 & 13.195 & 20.130 & 0.020 & Mar 11 & 6.869 & 20.478 & 0.024 & Mar 11 & 10.206 & 20.582 & 0.049 \\
\hline Jan 13 & 7.472 & 20.071 & 0.013 & Jan 13 & 13.269 & 20.159 & 0.020 & Mar 11 & 6.917 & 20.479 & 0.022 & Mar 11 & 10.254 & 20.610 & 0.053 \\
\hline Jan 13 & 7.546 & 20.098 & 0.013 & Jan 13 & 13.344 & 20.142 & 0.020 & Mar 11 & 6.966 & 20.460 & 0.023 & Mar 11 & 10.302 & 20.561 & 0.051 \\
\hline Jan 13 & 7.620 & 20.102 & 0.012 & Mar 11 & 2.850 & 20.446 & 0.033 & Mar 11 & 7.016 & 20.475 & 0.024 & Mar 11 & 10.354 & 20.610 & 0.054 \\
\hline
\end{tabular}


Table 2

(Continued)

\begin{tabular}{|c|c|c|c|c|c|c|c|c|c|c|c|c|c|c|c|}
\hline Date $^{a}$ & $\mathrm{UT}^{\mathrm{b}}$ & $m_{R}^{\mathrm{c}}$ & $\sigma_{m_{R}}{ }^{\mathrm{d}}$ & Date $^{\mathrm{a}}$ & $\mathrm{UT}^{\mathrm{b}}$ & $m_{R}{ }^{\mathrm{c}}$ & $\sigma_{m_{R}}{ }^{\mathrm{d}}$ & Date $^{a}$ & $\mathrm{UT}^{\mathrm{b}}$ & $m_{R}{ }^{\mathrm{c}}$ & $\sigma_{m_{R}}^{\mathrm{d}}$ & Date $^{\mathrm{a}}$ & $\mathrm{UT}^{\mathrm{b}}$ & $m_{R}^{\mathrm{c}}$ & $\sigma_{m_{R}}^{\mathrm{d}}$ \\
\hline Apr 4 & 3.028 & 20.766 & 0.044 & Apr 4 & 4.973 & 20.710 & 0.025 & Apr 4 & 7.177 & 20.829 & 0.031 & Apr 6 & 4.040 & 20.890 & 0.028 \\
\hline Apr 4 & 3.071 & 20.767 & 0.027 & Apr 4 & 5.021 & 20.732 & 0.026 & Apr 4 & 7.225 & 20.853 & 0.031 & Apr 6 & 4.090 & 20.926 & 0.033 \\
\hline Apr 4 & 3.127 & 20.775 & 0.028 & Apr 4 & 5.069 & 20.691 & 0.026 & Apr 4 & 7.275 & 20.795 & 0.031 & Apr 6 & 4.138 & 20.889 & 0.027 \\
\hline Apr 4 & 3.176 & 20.773 & 0.028 & Apr 4 & 5.117 & 20.657 & 0.025 & Apr 4 & 7.323 & 20.842 & 0.032 & Apr 6 & 4.186 & 20.859 & 0.026 \\
\hline Apr 4 & 3.224 & 20.794 & 0.028 & Apr 4 & 5.166 & 20.711 & 0.029 & Apr 4 & 7.371 & 20.794 & 0.033 & Apr 6 & 4.235 & 20.910 & 0.026 \\
\hline Apr 4 & 3.275 & 20.723 & 0.028 & Apr 4 & 5.220 & 20.719 & 0.028 & Apr 4 & 7.419 & 20.844 & 0.033 & Apr 6 & 4.283 & 20.917 & 0.026 \\
\hline Apr 4 & 3.323 & 20.706 & 0.025 & Apr 4 & 5.268 & 20.727 & 0.027 & Apr 4 & 7.468 & 20.833 & 0.033 & Apr 6 & 4.333 & 20.893 & 0.025 \\
\hline Apr 4 & 3.371 & 20.738 & 0.027 & Apr 4 & 5.316 & 20.708 & 0.027 & Apr 4 & 7.522 & 20.802 & 0.034 & Apr 6 & 4.381 & 20.855 & 0.024 \\
\hline Apr 4 & 3.420 & 20.776 & 0.028 & Apr 4 & 5.364 & 20.713 & 0.027 & Apr 4 & 7.570 & 20.830 & 0.036 & Apr 6 & 4.429 & 20.858 & 0.024 \\
\hline Apr 4 & 3.468 & 20.758 & 0.029 & Apr 4 & 5.412 & 20.689 & 0.026 & Apr 4 & 7.618 & 20.820 & 0.037 & Apr 6 & 4.478 & 20.857 & 0.024 \\
\hline Apr 4 & 3.517 & 20.722 & 0.028 & Apr 4 & 5.468 & 20.735 & 0.027 & Apr 4 & 7.666 & 20.745 & 0.037 & Apr 6 & 4.526 & 20.862 & 0.025 \\
\hline Apr 4 & 3.566 & 20.772 & 0.027 & Apr 4 & 5.716 & 20.731 & 0.026 & Apr 4 & 7.715 & 20.824 & 0.042 & Apr 6 & 4.575 & 20.854 & 0.025 \\
\hline Apr 4 & 3.614 & 20.724 & 0.025 & Apr 4 & 5.781 & 20.739 & 0.026 & Apr 4 & 7.764 & 20.766 & 0.042 & Apr 6 & 4.624 & 20.826 & 0.025 \\
\hline Apr 4 & 3.662 & 20.764 & 0.027 & Apr 4 & 5.829 & 20.732 & 0.026 & Apr 4 & 7.812 & 20.797 & 0.037 & Apr 6 & 4.672 & 20.821 & 0.027 \\
\hline Apr 4 & 3.711 & 20.759 & 0.026 & Apr 4 & 5.878 & 20.760 & 0.026 & Apr 4 & 7.860 & 20.804 & 0.036 & Apr 6 & 4.855 & 20.829 & 0.033 \\
\hline Apr 4 & 3.760 & 20.769 & 0.026 & Apr 4 & 5.926 & 20.782 & 0.026 & Apr 4 & 7.908 & 20.835 & 0.038 & Apr 6 & 4.952 & 20.811 & 0.030 \\
\hline Apr 4 & 3.808 & 20.735 & 0.026 & Apr 4 & 5.974 & 20.769 & 0.026 & Apr 4 & 7.957 & 20.814 & 0.038 & Apr 6 & 5.243 & 20.747 & 0.028 \\
\hline Apr 4 & 3.857 & 20.750 & 0.025 & Apr 4 & 6.023 & 20.758 & 0.027 & Apr 4 & 8.006 & 20.855 & 0.037 & Apr 6 & 5.291 & 20.763 & 0.028 \\
\hline Apr 4 & 3.905 & 20.728 & 0.026 & Apr 4 & 6.072 & 20.784 & 0.026 & Apr 4 & 8.054 & 20.839 & 0.038 & Apr 6 & 5.341 & 20.740 & 0.027 \\
\hline Apr 4 & 3.953 & 20.784 & 0.027 & Apr 4 & 6.120 & 20.790 & 0.027 & Apr 4 & 8.102 & 20.773 & 0.034 & Apr 6 & 5.389 & 20.794 & 0.029 \\
\hline Apr 4 & 4.002 & 20.714 & 0.028 & Apr 4 & 6.168 & 20.764 & 0.026 & Apr 4 & 8.151 & 20.781 & 0.035 & Apr 6 & 5.437 & 20.745 & 0.036 \\
\hline Apr 4 & 4.051 & 20.756 & 0.028 & Apr 4 & 6.216 & 20.786 & 0.026 & Apr 4 & 8.199 & 20.797 & 0.037 & Apr 6 & 5.486 & 20.743 & 0.031 \\
\hline Apr 4 & 4.099 & 20.758 & 0.026 & Apr 4 & 6.305 & 20.790 & 0.026 & Apr 4 & 8.250 & 20.737 & 0.036 & Apr 6 & 5.534 & 20.743 & 0.025 \\
\hline Apr 4 & 4.147 & 20.710 & 0.025 & Apr 4 & 6.354 & 20.805 & 0.027 & Apr 4 & 8.298 & 20.763 & 0.038 & Apr 6 & 5.583 & 20.735 & 0.025 \\
\hline Apr 4 & 4.195 & 20.724 & 0.026 & Apr 4 & 6.402 & 20.795 & 0.028 & Apr 4 & 8.346 & 20.741 & 0.038 & Apr 6 & 5.631 & 20.696 & 0.024 \\
\hline Apr 4 & 4.244 & 20.744 & 0.028 & Apr 4 & 6.450 & 20.819 & 0.028 & Apr 4 & 8.394 & 20.750 & 0.053 & Apr 6 & 5.679 & 20.741 & 0.025 \\
\hline Apr 4 & 4.292 & 20.710 & 0.025 & Apr 4 & 6.498 & 20.817 & 0.028 & Apr 4 & 8.442 & 20.754 & 0.045 & Apr 6 & 5.728 & 20.731 & 0.025 \\
\hline Apr 4 & 4.341 & 20.719 & 0.024 & Apr 4 & 6.547 & 20.823 & 0.029 & Apr 4 & 8.498 & 20.798 & 0.051 & Apr 6 & 5.776 & 20.716 & 0.025 \\
\hline Apr 4 & 4.389 & 20.716 & 0.024 & Apr 4 & 6.595 & 20.850 & 0.030 & Apr 4 & 8.546 & 20.756 & 0.048 & Apr 6 & 5.825 & 20.707 & 0.025 \\
\hline Apr 4 & 4.438 & 20.684 & 0.026 & Apr 4 & 6.644 & 20.851 & 0.029 & Apr 4 & 8.594 & 20.751 & 0.049 & Apr 6 & 5.873 & 20.705 & 0.024 \\
\hline Apr 4 & 4.488 & 20.698 & 0.023 & Apr 4 & 6.692 & 20.835 & 0.029 & Apr 4 & 8.642 & 20.699 & 0.050 & Apr 6 & 5.921 & 20.719 & 0.025 \\
\hline Apr 4 & 4.536 & 20.687 & 0.023 & Apr 4 & 6.740 & 20.810 & 0.030 & Apr 4 & 8.740 & 20.729 & 0.055 & Apr 6 & 5.970 & 20.704 & 0.026 \\
\hline Apr 4 & 4.584 & 20.712 & 0.023 & Apr 4 & 6.790 & 20.855 & 0.031 & Apr 6 & 3.029 & 20.856 & 0.134 & Apr 6 & 6.018 & 20.728 & 0.027 \\
\hline Apr 4 & 4.632 & 20.724 & 0.023 & Apr 4 & 6.839 & 20.817 & 0.031 & Apr 6 & 3.646 & 20.858 & 0.027 & Apr 6 & 6.067 & 20.724 & 0.027 \\
\hline Apr 4 & 4.680 & 20.719 & 0.023 & Apr 4 & 6.887 & 20.850 & 0.032 & Apr 6 & 3.701 & 20.865 & 0.027 & Apr 6 & 6.115 & 20.727 & 0.027 \\
\hline Apr 4 & 4.730 & 20.727 & 0.024 & Apr 4 & 6.935 & 20.894 & 0.033 & Apr 6 & 3.749 & 20.920 & 0.027 & Apr 6 & 6.164 & 20.725 & 0.027 \\
\hline Apr 4 & 4.779 & 20.699 & 0.025 & Apr 4 & 6.983 & 20.877 & 0.031 & Apr 6 & 3.797 & 20.875 & 0.027 & Apr 6 & 6.212 & 20.715 & 0.029 \\
\hline Apr 4 & 4.827 & 20.672 & 0.026 & Apr 4 & 7.032 & 20.824 & 0.029 & Apr 6 & 3.847 & 20.888 & 0.025 & Apr 6 & 6.260 & 20.710 & 0.028 \\
\hline Apr 4 & 4.875 & 20.684 & 0.025 & Apr 4 & 7.080 & 20.867 & 0.030 & Apr 6 & 3.895 & 20.901 & 0.027 & & & & \\
\hline Apr 4 & 4.923 & 20.711 & 0.025 & Apr 4 & 7.129 & 20.859 & 0.031 & Apr 6 & 3.992 & 20.901 & 0.028 & & & & \\
\hline
\end{tabular}

Notes.

${ }^{\text {a }}$ UT date of observations.

${ }^{\mathrm{b}}$ UT at midpoint of the exposure (uncorrected for light travel time).

c Observed $R$-band magnitude (after applying absolute calibrations, extinction corrections, and comparison star corrections).

${ }^{\mathrm{d}}$ Uncertainty in the observed magnitude.

minimum varied in depth and shape with changing viewing geometry. Unfortunately, our phase coverage is incomplete in April and the shallower minimum is not sampled, so we cannot determine if the change in depth from January to March continued. Also evident is a $\sim 0.02 \mathrm{mag}$ shift of much (but not all) of the January 12 lightcurve from that of a day later on January 13, for which we have been unable to identify a cause. While this has no effect on the period solution, it alters the perception of the shape for the first half of the lightcurve.

We also phased subsets of the data to look for evidence of a change in the observed period associated with the changing viewing geometries. As expected, phasing January/April yielded the same result as the entire data set, while a minor change was evident for the other two pairings-January/March gave a slightly smaller value $(8.947 \pm 0.001 \mathrm{hr})$ and March/ April gave a slightly larger value $(8.949 \pm 0.002 \mathrm{hr})$ with the higher uncertainty in the latter value directly caused by the shorter interval. Within the uncertainties, this increase matches the predicted change of $+0.001 \mathrm{hr}$, caused simply by Earth's orbital motion between our earliest midpoint pairing and the latest; the solar component to the synodic period near aphelion is both small and unchanging. These calculations all use the pole orientation from Knight et al. (2012) of R.A. $=162^{\circ}$ and decl. $=+58^{\circ}$ (and the diametrically opposite solution for retrograde rotation), but similar pole positions, such as from Sekanina (1991), would yield the same results.

As discussed in the Introduction, one goal of these observations was to uniquely discriminate between prograde and retrograde rotation for Tempel 2's nucleus. The sense of rotation always determines the sign of the solar component of the synodic period, while Earth's component is unaffected by the direction of rotation. At the midpoint of our ensemble of measurements 
Table 3

Sidereal Rotation Periods and Lightcurve Amplitudes

\begin{tabular}{|c|c|c|c|c|c|c|}
\hline \multirow[t]{2}{*}{$\mathrm{UT}_{\text {date }}^{\mathrm{a}}$} & \multirow{2}{*}{$\begin{array}{c}\text { Synodic } \\
\text { Period (hr) }\end{array}$} & \multicolumn{2}{|c|}{ Sidereal Period $(\mathrm{hr})^{\mathrm{b}}$} & \multirow{2}{*}{$\begin{array}{c}\text { Amplitude } \\
\text { (mag) }\end{array}$} & \multirow{2}{*}{$\begin{array}{c}\text { Sub-Earth } \\
\text { Latitude }\left(^{\circ}\right)\end{array}$} & \multirow{2}{*}{$\begin{array}{l}\text { Sub-solar } \\
\text { Latitude }\left(^{\circ}\right)\end{array}$} \\
\hline & & Prograde $^{c}$ & Retrograde & & & \\
\hline 1987 Apr 1 & $\cdots$ & $\ldots$ & $\ldots$ & 0.3 & -52 & -46 \\
\hline 1988 Apr 28 & $8.932 \pm 0.001$ & 8.931 & 8.935 & $0.5-0.8$ & -2 to -10 & -18 to +17 \\
\hline 1994 Nov 22 & $8.939 \pm 0.003$ & 8.939 & 8.941 & 0.5 & -3 to +4 & +2 to -7 \\
\hline 1999 May 20 & $8.941 \pm 0.002$ & 8.939 & 8.945 & $0.5-0.7$ & +9 to +10 & -1 to +21 \\
\hline 2010 Oct 25 & $8.950 \pm 0.002$ & 8.948 & 8.955 & $0.5-0.8$ & +35 to +43 & +40 to +13 \\
\hline 2013 Feb 24 & $8.948 \pm 0.001$ & 8.948 & 8.950 & 0.2 & -47 & -48 \\
\hline
\end{tabular}

Notes.

a Midpoint of the observations used to determine the synodic period. The relevant perihelion dates are 1988 September 16 , 1994

March 16, 1999 September 8, 2005 February 15, 2010 July 4, and 2015 November 14.

b The estimated uncertainties are identical to those listed in Column 2.

c The combined 2010 and 2013 results reveal that Tempel 2 is in prograde rotation.

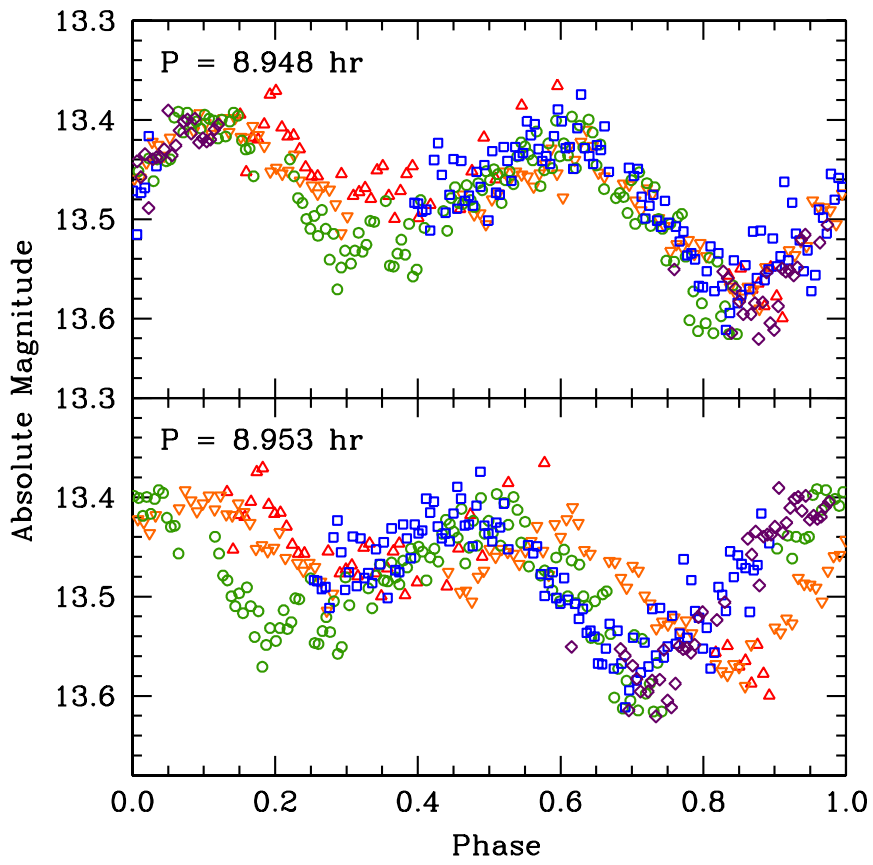

Figure 2. Lightcurve data phased to $8.948 \mathrm{hr}$ (top) and $8.953 \mathrm{hr}$ (bottom). The symbols are as given in Figure 1 and the magnitudes are normalized to $(1,1,0)$ as described in the text. The $8.948 \mathrm{hr}$ period is the synodic period expected in 2013 based on the 2010 prograde sidereal solution found in Knight et al. (2012) while the $8.953 \mathrm{hr}$ period is the synodic period expected in 2013 based on the 2010 retrograde sidereal solution. The $8.953 \mathrm{hr}$ period is clearly incompatible with the 2013 data, thus definitively ruling out retrograde rotation.

for 2013, the solar and Earth components are coincidentally nearly identical and in the prograde case have opposite signs that cancel out, resulting in the sidereal period being the same as the synodic $(8.948 \pm 0.001 \mathrm{hr})$, while in the retrograde case they compound yielding a sidereal value of $8.950 \pm 0.001 \mathrm{hr}$.

To determine which solution is correct, we next reexamine the solutions from 2010. Our overall observed (synodic) value was $8.950 \pm 0.002 \mathrm{hr}$ with a corresponding midpoint time of 2010 October 25 or 112 days following perihelion (Knight et al. 2012). Based on our water production rates throughout the apparition, over $95 \%$ of the total outgassing had taken place by this date. With an observed change in period of about $+0.004 \mathrm{hr}$ per perihelion passage, we concluded that further torquing late in the 2010 apparition should be negligible and that the sidereal period in 2013 would therefore be unchanged from the late 2010 result. The corresponding sidereal values at the 2010 midpoint are $8.948 \mathrm{hr}$ (prograde) and $8.955 \mathrm{hr}$ (retrograde). Note that these values are slightly different from those listed in Knight et al. (2012) which erroneously only included the solar component but not Earth's component. With the expectation that the sidereal value should be the same in early 2013 as in late 2010, and the possible sidereal values in 2013 being $8.948 \pm 0.001 \mathrm{hr}$ (prograde) and $8.950 \pm 0.001 \mathrm{hr}$ (retrograde), we conclude that Tempel 2 must be in prograde rotation, with a sidereal period in late 2010 and in early 2013 of $8.948 \mathrm{hr}$.

As further evidence that Tempel 2 is in a prograde rotation, we also examined the retrograde scenario. If it was the retrograde case, then as already stated the sidereal period in 2010 would have been $8.955 \mathrm{hr}$. Since the difference between the retrograde synodic and sidereal periods in 2013 would be $-0.002 \mathrm{hr}$, we should have measured a period of $8.953 \mathrm{hr}$. Phasing with this value is shown in the bottom panel of Figure 2 and it is clear that this solution does not yield a viable lightcurve; therefore, the comet must instead be in prograde rotation.

Looking back at the earlier apparitions requires disentangling the synodic effects from the actual changes in period due to torquing. An early attempt to derive the sidereal period from the changing synodic period during 1988 (Sekanina 1991) suffered from several problems, including the claim of a smoothly decreasing period which could not be reproduced during a detailed reanalysis (Knight et al. 2011) and our new determination that his synodic/sidereal modeling only included Earth's motion but neglected the dominant solar component. Having now definitively determined the sense of rotation as prograde, we can reexamine prior apparitions. The computed sidereal periods for each epoch are given in Table 3; for completeness and to illustrate the asymmetries of the proand retrograde solutions with respect to the observed synodic periods, we also tabulate the retrograde values. Again, these values differ somewhat from the values given in Knight et al. (2012) since the Earth component is now included along with the solar component, thereby causing the asymmetry. Our conclusions regarding a decrease in the amount of spin-down remain unchanged from Knight et al. (2012), with sidereal periods before perihelia in 1988 of $8.931 \mathrm{hr}$ and in 1999 of $8.939 \mathrm{hr}$, for an average spin-down by $0.004 \mathrm{hr}$ per apparition. The same rate of spin-down would have predicted a sidereal period of $8.951 \mathrm{hr}$ in late 2010, a larger change than we observe. We continue to think the most likely cause of this is the decrease in total water production from 1988 to 1999 and to 2010, with lower production rates providing a smaller amount of torquing. However, within the uncertainties of the period determinations, 
the data are also consistent with no change in the rate of spin-down. The agreement between the late 1994 period with early 1999 also remains, with both yielding a sidereal period of $8.939 \mathrm{hr}$ during an interval for which there was not an intervening perihelion passage.

\subsection{Nucleus Cross-section}

Our 2013 lightcurves of Tempel 2 revealed two unexpected findings. First, the amplitude within the lightcurve was smaller than we had assumed it would be and, second, the peak brightness was higher than predicted from earlier apparitions even after compensating for a non-linear phase function. After considering a variety of possibilities, such as removing too much or too little coma for differing apparitions or systematic effects when different filters were used, we think we have arrived at a self-consistent explanation. Our expectations for 2013 were based on assuming that Tempel 2 is a prolate ellipsoid with similar dimensions for the small and intermediate axes while the long axis is about $2.1 \times$ greater. This is based on the 1988 thermal IR lightcurve from A'Hearn et al. (1989) and that the sub-Earth latitude then varied between $-1^{\circ}$ and $-9^{\circ}$, i.e., the comet was viewed in 1988 nearly equator-on using our preferred pole solution. In early 2013, the sub-Earth latitude was $-47^{\circ}$, yielding a predicted amplitude of $0.4 \mathrm{mag}$, significantly greater than the observed value of $\sim 0.2 \mathrm{mag}$. If, however, the intermediate axis is actually intermediate in length rather than the same as the small axis, then the total cross-section will appear to increase as one views from more pole-on and the amplitude will become even smaller than the simple function of the cosine of the sub-Earth latitude (the intermediate axis begins to dominate over the small axis). Note that in this tri-axial case, it is the ratio of the long axis to the intermediate axis that determines the amplitude for the equator-on view. Therefore, to explain the 1988 amplitude, the intermediate axis remains the same as originally assumed for the prolate case, and it is the short axis which must be even shorter. For our observed amplitude in 2013, this requires the short axis to be less than one-third that of long axis, while the intermediate axis remains at about one-half of the long axis. This implies an even more elongated nucleus than the tri-axial solution with ratios of 0.43:0.60:1.0 proposed by Sekanina (1991). In addition to explaining the observed amplitude, our tri-axial solution also naturally explains the increased brightness at lightcurve maxima in 2013 as being due to the increased total cross-section relative to the equator-on view.

We strongly suspect, however, that the shape of the nucleus significantly departs from a tri-axial ellipsoid for several reasons. The lightcurve shape is not sinusoidal, but rather has one "V"-shaped minimum and one rounded minimum. Also, the shapes and depths of the minima change rapidly in 2013 with only a small change in viewing geometries even though the subEarth and sub-solar latitudes did not vary. Finally, as discussed below, the amplitudes are quite different when the comet was viewed from high positive latitudes as compared to high negative latitudes. Thus, we conclude that the nucleus must have large-scale protuberances.

To first-order, the tri-axial ellipsoid explains most of the brightness and amplitudes measured at the other apparitions. Most similar to 2013 were the circumstance in early 1987 with a sub-Earth latitude of $-52^{\circ}$, and where Jewitt \& Meech (1988) measured an amplitude of $\sim 0.3 \mathrm{mag}$ and a peak magnitude essentially identical to our 2013 value once adjustments are made for filter and phase angle. The 1999 apparition was nearly identical to that of 1988, and the lightcurve characteristics are also essentially the same. The peak brightness in 1994 (Mueller \& Ferrin 1996) is also consistent with 1988 and 1999 but the amplitude is somewhat smaller (by $\sim 0.1-0.2 \mathrm{mag}$ ) with no clear explanation since the sub-Earth latitude was close to the equator. Most different were our results from 2010 (Knight et al. 2012), where the peak brightness is $\sim 0.2-0.3$ mag fainter while the amplitude is larger than expected for a sub-Earth latitude near $+40^{\circ}$. While an over removal of coma might partially explain both aspects, our methodology was the same used for the 1999 apparition which has no such issues, leading us to suspect that Tempel 2's actual shape characteristics are another significant factor; this is supported by the fact that only in 2010 did the subEarth latitude have a high positive value $\left(+40^{\circ}\right)$ while all other apparitions were either near the equator $(1988,1994,1999)$ or at high negative values $(1987,2013)$.

\section{DISCUSSION AND SUMMARY}

The observations reported here represent some of the first science collected with Lowell Observatory's new Discovery Channel Telescope. Despite a few problems associated with a facility still in the early stages of commissioning, we were able to easily fulfill our major science objectives regarding Comet Tempel 2's rotational state, in spite of a smaller lightcurve amplitude than expected. We obtained a precise measurement of its current rotation period that, when combined with our 2010 measurements, yielded a definitive determination of its sense of rotation. This result in turn allowed us to determine the correct sidereal period associated with observed synodic values at each prior apparition. For Lowell Observatory and its partners, a new era has begun in which projects requiring time-intensive or long-duration observations can be accomplished for targets much fainter than have ever been possible. In the specific case of comet nuclei, we can now measure lightcurves of comet nuclei far from perihelion.

The measured periodicity in a nucleus lightcurve depends on the changing cross-section both as illuminated by the Sun and as seen from the Earth, and in turn depends on the solar day as well as the Earth's motion with respect to the comet and the Sun. Disentangling these effects from the change in the physical rotation due to torquing had been problematic at best. However, the relative importance of the solar component and Earth's motion component are quite different far from the Sun as compared to near perihelion. Because of this, we planned and executed these new observations. The resulting synodic period, $8.948 \pm 0.001 \mathrm{hr}$, matched that expected for the prograde scenario from 2010 while the nominal period associated with the retrograde solution is clearly ruled out. We therefore conclude that Tempel 2's rotation is prograde with respect to both the ecliptic and to its orbital plane. The sidereal period, $8.948 \pm 0.001 \mathrm{hr}$, is coincidentally identical to the synodic value as the solar and Earth components of the synodic period just cancel out during the interval of our 2013 observations. The new data also confirm that no additional spin-down took place following our late 2010 measurements, consistent with the water production curve showing a near-cessation of activity by that time. This lack of a change in the sidereal period very late in the 2010 apparition thereby implies that the smaller change in the period per perihelion passage between 1999 and 2010 as compared to 1988 to 1999 is real (although a constant rate of spin-down cannot be excluded within the uncertainties). Note that the inferred changes match those given by Knight et al. (2012) for the prograde case. Thus our hypothesis that 
a decrease in torquing is associated with the secular decrease in water production from 1988 to 2010 is probably correct. We note, however, that there is no strong evidence for a long-term decrease in activity over the past century, and the secular drop over the past two decades may reverse as the surface is eroded and varying proportions of ice are exposed from successive perihelion passages.

Similar to prior apparitions, the light curve is double-peaked with near-equal maxima but quite differently shaped minima. The shape and depth of one of the minima also changed over the three-month interval, another trait observed in the past. Both characteristics directly indicate that the shape of Tempel 2's nucleus is not a simple prolate ellipsoid or even a tri-axial ellipsoid but must instead have large-scale protuberances to cause such clear changes in the lightcurve from such a small change in viewing geometries $\left(<20^{\circ}\right)$ and essentially no change in sub-Earth and sub-solar latitudes. The unexpectedly small amplitude we measured in the lightcurve $(\sim 0.2 \mathrm{mag})$ appears to also be due to peculiarities of the nucleus shape-our value is in good agreement with the only other data obtained at a similar sub-Earth latitude (Jewitt \& Meech 1988), while data taken from near-equator on or from the other hemisphere all exhibit a much larger amplitude. The data further suggest that the length of the short axis is substantially shorter than that of the intermediate axis, though the shape is probably not as extreme as that of Comet 103P/Hartley 2 as imaged from EPOXI (A'Hearn et al. 2011). Thus a more complete story of the properties of Tempel 2's nucleus is beginning to emerge. While determining the detailed shape must continue to await an upclose view-Tempel 2 was the proposed target of the planned Comet Rendezvous Asteroid Flyby mission more than two decades ago - additional numerical modeling and new investigations from afar can continue to reveal clues to its physical structure and evolving behavior.

We thank T. Farnham for calculations of the offsets between the prograde and retrograde synodic periods with respect to a given sidereal period. We gratefully acknowledge the assistance of $\mathrm{M}$. Fendrock with the first observing run and preliminary analyses, and A. Venetiou, M. Sweaton, J. Sanborn, R. Winner, and S. Strosahl for their successful operations during the early commissioning phase of the DCT, thereby making these observations possible. We also thank R. Millis, W. L. Putnam, and J. Hendricks for their vision and support which allowed the DCT to become a reality, and all members of the DCT and LMI teams.

These results made use of Lowell Observatory's Discovery Channel Telescope, supported by Lowell, Discovery Communications, Boston University, the University of Maryland, and the University of Toledo. M.M.K. is grateful for office space provided by the University of Maryland Department of Astronomy and Johns Hopkins University Applied Physics Laboratory while working on this project. The LMI instrument was funded by the National Science Foundation via grant AST-1005313. This research has been supported by NASA's Planetary Astronomy Program (grant NNX09AB51G).

\section{REFERENCES}

A'Hearn, M. F., Belton, M. J. S., Delamere, W. A., et al. 2011, Sci, 332, 1396

A'Hearn, M. F., Campins, H., Schleicher, D. G., \& Millis, R. L. 1989, ApJ, 347,1155

Bida, T. A., Dunham, E. W., Bright, L. P., \& Corson, C. 2004, Proc. SPIE, 5489, 196

Bida, T. A., Dunham, E. W., Nye, R. A., Chylek, T., \& Oliver, R. C. 2012, Proc. SPIE, 8444, 844451

Bowell, E., \& Lumme, K. 1979, in Asteroids, ed. T. Gehrels (Tucson, AZ: Univ. of Arizona Press), 132

Jewitt, D., \& Luu, J. 1989, AJ, 97, 1766

Jewitt, D. C., \& Meech, K. J. 1988, ApJ, 328, 974

Knight, M. M., Farnham, T. L., Schleicher, D. G., \& Schwieterman, E. W. 2011, AJ, 141, 2

Knight, M. M., Schleicher, D. G., Farnham, T. L., Schwieterman, E. W., \& Christensen, S. R. 2012, AJ, 144, 153

Levine, S. E., Bida, T. A., Chylek, T., et al. 2012, Proc. SPIE, 8444, 844419

Massey, P., Dunham, E. W., Bida, T. A., et al. 2013, AAS Meeting \#221, poster \#345.02

Mueller, B. E. A., \& Ferrin, I. 1996, Icar, 123, 463

Sekanina, Z. 1979, Icar, 37, 420

Sekanina, Z. 1991, AJ, 102, 350

Stellingwerf, R. F. 1978, ApJ, 224, 953

Wisniewski, W. Z. 1990, Icar, 86, 52 